\title{
Familia, género y espacio transnacional en Dime algo sobre Cuba, de Jesús Díaz
}

\author{
Fredrik Olsson (University of Gothenburg / Linnaeus University, \\ Sweden)
}

\begin{abstract}
Family and intimate relationships across borders is a central topic in migration literature. This article investigates the representation of the transnational family in Dime algo sobre Cuba (1998), by the Cuban writer and filmmaker Jesús Díaz. Written from exile in Spain, the novel is set in Cuba's "Special Period" of post-Cold War economic crisis and emigration of balseros ('rafters'). The highly original plot of a twofold perilous voyage between Havana and Miami incorporates elements from both exile literature and undocumented migration narratives, but it also goes beyond the established patterns of these genres. Drawing on transnational family studies and feminist theory, this paper examines how the characters experience the migration process with focus on the internal dynamics of the subjects that comprise the family, their relations to multiple places, as well as the narrative modes of representing these relations. It shows the internal dynamics of the protagonist's family as a split narrative of disand reintegration across political and national borders. It also discusses the lived experience of the double orientation of the migrant subject, facing a lost home(land) as well as a new place which s/he still does not inhabit. The analysis suggests that the process of the reorientation of the migrant subject is articulated as a gendered and sexualised narrative of the intimate relations of the protagonist, intertwined with the narrative of the homeland. However, the ambivalent ending of the novel with its references to cultural hybridity points to an opening where the future of Cuban exile and diaspora lies in the ability to forgive and establish cultural contact across borders.
\end{abstract}

Keywords: migration, Cuba, transnational family, borders, Jesús Díaz

\section{Introducción}

Las relaciones familiares e íntimas a través de las fronteras —nacionales, culturales, sociales, políticas, lingüísticas, de género y generación — es una temática central en la literatura de la migración. Ya en la literatura clásica y obras como la Odisea, de Homero, se encuentran narrativas de des- y reintegración familiar por exilio. Ahora bien, aunque es posible observar la persistencia de ciertos temas recurrentes tales como el mito de la tierra prometida (Aínsa 1988), también es preciso reconocer la especificidad de cada migración. Así pues, la representación literaria de la inmigración hispana en Estados Unidos comprende la narración de experiencias e imaginarios variados según el contexto histórico, político y generacional, entendido este 
último término tanto en el sentido de relaciones intrafamiliares, biológicamente basadas, como en el sentido de generaciones histórico-políticas (Eckstein y Barberia 2002, 800-801).

Este trabajo se centra en la representación de la familia transnacional en Dime algo sobre Cuba (1998), de Jesús Díaz. Después de una larga carrera como escritor, editor y cineasta dentro de Cuba y la Revolución, Díaz escribió Dime algo sobre Cuba desde el exilio en Madrid, siendo esta la última etapa de la evolución de su producción literaria, que se caracterizó por el movimiento "de la ilusión a la desilusión" (Guerrero 2002, 16). Desde los años 1960, cuando Díaz llegó a ser una figura central de la cultura cubana con la obtención del premio Casa de las Américas por la colección de cuentos Los años duros (1966) y el trabajo como editor de la revista cultural El Caimán Barbudo, ${ }^{1}$ había sido una voz disonante, pero fiel a la Revolución. Optando por el exilio en Europa en 1992, reinventó su misión cultural con la fundación de la revista disidente Encuentro de la Cultura Cubana en Madrid, que tenía como misión el debate, el diálogo y la reconciliación de la nación y la cultura cubanas de dentro y fuera (Grenier 2017; Rojas 2006, 317-320).

En Dime algo sobre Cuba, la trama transcurre entre la Cuba revolucionaria de los años 1990 y la comunidad cubana en Florida. Este "período especial" se caracterizó por el deterioro de la economía y la extrema escasez después de la caída del muro de Berlín, lo que llevó a un desencanto entre muchos intelectuales que previamente habían apoyado la Revolución, así como una salida masiva de "balseros", es decir, migrantes que al llegar a la costa o al agua territorial de Estados Unidos eran otorgados asilo político por la Ley de Ajuste Cubano (Eckstein y Barberia 2002, 806; Sánchez Becerril 2012, 86-87). La novela narra el proceso a través del que el protagonista, el dentista habanero con el nombre significativo Stalin Martínez, deja su país, su trabajo y a su (ex)mujer para convertirse en falso balsero e indocumentado en Miami. Allí se refugia en la azotea de la casa familiar de su hermano Lenin, que ha venido una década atrás en el éxodo por el puerto de Mariel en 1980 en la que más de cien mil personas emigraron a Estados Unidos, y conoce a la joven cubano-americana Miriam.

A diferencia de otros textos ficcionales de balseros y otros exiliados del período especial, ${ }^{2}$ Dime algo sobre Cuba se sitúa, pues, en la intersección entre exilio, diáspora y migración indocumentada. Díaz cruza en un mismo texto dos de las posiciones textuales principales de la literatura hispana de Estados Unidos trazadas por Kanellos (2011, 19-34), a saber, la de exilio y la de inmigración. El protagonista, refugiado tanto en el sentido político como en el sentido económico, aúna la experiencia de balsero con la del migrante indocumentado que viaja a Estados Unidos por tierra. Este nexo es algo insólito en las letras hispanoamericanas, y también distingue la novela de las otras de Díaz de temática migratoria, ${ }^{3}$ pues agrega rasgos del subgénero de la literatura de la migración indocumentada de América Latina (en particular, México) a Estados Unidos ( $c f$. Olsson 2016) en una trama singular que

\footnotetext{
${ }^{1}$ El Caimán Barbudo era el suplemento cultural del periódico Juventud Rebelde, publicado por la Unión de Jóvenes Comunistas de Cuba.

${ }^{2}$ Ejemplos de otros escritores que han retratado el período especial y la salida al extranjero son Zoé Valdés, Abilio Estévez (desde el exilio en Europa) y Leonardo Padura (desde la isla); de la generación más joven —los novísimos (cf. Sánchez Becerril 2012) — destacan, por ejemplo, Ena Lucía Portela y Wendy Guerra, con la novela Todos se van (2006). Cuentos de todas partes del imperio (2000), de Antonio José Ponte, es una interesante muestra de la representación de cubanos en el mundo.

${ }^{3}$ Ejemplos son La piel y la máscara (1996), que se centra en las relaciones transnacionales entre Cuba y Miami, y Siberiana (2000), que trata de las aventuras de un joven reportero cubano en Siberia. Las cuatro fugas de Manuel (2002) narra el cruce de múltiples fronteras del protagonista y sus problemas con las autoridades migratorias, pero está ambientada en Europa en la época de la caída de la Unión Soviética.
} 
resulta de particular interés para el estudio de la representación de la migración transnacional y su impacto sobre la familia y los sujetos que la componen.

A pesar de esto, en el campo de los estudios de Díaz, Dime algo sobre Cuba ocupa un lugar algo marginal en comparación con algunas de sus obras más conocidas como Las iniciales de la tierra (1987) y Las palabras perdidas (1992), ubicadas más firmemente en Cuba y los años tempranos de la Revolución. En una investigación clave sobre los mecanismos del discurso utópico en la Cuba revolucionaria, Gustafsson (2014) analiza Dime algo sobre Cuba a partir de los conceptos de memoria y migración y en comparación con otras narrativas ficcionales de la época como La nada cotidiana (1995) de Zoé Valdés, y la película Fresa y chocolate (1993) de Tomas Alea Gutiérrez. Ignizio (2016), por su parte, se centra en la representación del período especial a través del imaginario de la comida. El presente trabajo, aunque se nutre de estos y otros estudios de la literatura del período especial y el exilio cubano, toma un rumbo diferente y parte de la categoría de la familia para estudiar con mayor atención la configuración literaria del proceso de la migración.

Quisiera proponer la narrativa de la familia transnacional como un objeto de estudio propicio para explorar cómo se negocia la identidad entre, por un lado, los procesos culturales y las estructuras sociales, políticas y económicas a nivel macro y, por otro, las relaciones más íntimas de la familia ( $c f$. Bryceson y Vuorela 2002, 6, Dreby y Adkins 2010, 674). En el presente estudio se parte de la concepción de la familia como "una construcción simbólica a través de las narrativas elaboradas por sus miembros" (Martínez Ruiz y Reyes Tovar 2017, 117). En paralelismo con el concepto de la nación planteada por Anderson (2006 [1983]), y según la analogía entre familia y nación que tradicionalmente se ha establecido tanto en la antropología (Bryceson y Vuorela 2002, 9) como en la literatura (Sommer 1991), podemos entender la familia como una comunidad real e imaginada, es decir, una construcción social y cultural, siempre y cuando se tiene en cuenta que a diferencia de la nación, de la que el individuo no tiene una experiencia directa, la familia es una unidad pequeña y diferenciada, incluso cuando se encuentra espacialmente dispersa (Bryceson y Vuorela 2002, 10, Goulborne et al. 2010, 6).

Así las cosas, en el presente artículo se examinará cómo los personajes perciben y experimentan el proceso migratorio en la novela de Díaz, con el centro de atención en la dinámica interna de los sujetos que componen la familia y su relación con los lugares habitados o transitados, así como los modos para narrar estas relaciones. Se estudiará también la negociación de los sujetos entre moverse y morar y su orientación hacia el pasado, el presente y el futuro. Argüiré que la novela incorpora elementos tanto de la literatura de exilio como la literatura hispanoamericana de la migración indocumentada a Estados Unidos, pero que también sale de los patrones establecidos a través del itinerario singular del protagonista en el espacio transnacional entre La Habana y Miami, trazando una trama insólita en la intersección entre familia, nación y género. Después de estudiar las formas de narrar la familia, se explorarán los procesos de des- y reunificación familiar a través de las fronteras. En el último apartado, se discutirá la doble orientación del sujeto migrante y el papel de la familia y las relaciones íntimas en la narración de los procesos identitarios relacionados con la migración y el exilio.

\section{La narrativa escindida de la familia}

"'No quiero volver'", concluye el protagonista el día que despierta en la azotea de la casa de su hermano en Miami, y la voz narradora añade: "Fue entonces cuando decidió 
reconstruir con todo detalle la inextricable suma de acontecimientos que lo había conducido hasta allí, a ver si así lograba entender a la suerte y sobre todo entenderse a sí mismo" (Díaz 1998, 15). Desde el presente narrativo en la azotea, donde, según el plan ingeniado por su hermano, se está mortificando en el sol para pasar por balsero auténtico, es decir, alguien que tiene derecho al asilo político, Stalin comienza a reconstruir el proceso migratorio. El anónimo narrador externo enmarca ambos niveles narracionales, otorgando a la historia cierta objetividad e insertando una distancia, a veces con la ironía de la parodia, con respecto al protagonista. De acuerdo con Gustafsson $(2014,169)$, la azotea en Florida se configura como un no-lugar, un espacio absurdo e irreal y a la vez lo único real en el presente del migrante. Este lugar sirve en primer lugar para recordar, pues, como Stalin se pregunta: “¿Qué otra cosa podía hacer en aquella azotea?" (Díaz 1998, 25). Para el protagonista, el proceso mnemónico tiene la función de intentar entender los sucesos que le han llevado a Miami, pero también sirve para continuar el viaje geográfico e interior que ha iniciado al salir del país. Kaminsky $(1999,98)$ describe el exilio como el intento de "dar significado" (making meaning) en el lugar de acogida en relación con el lugar de origen: "The tension between within and without, the impossibility of making coherent meaning between one place and the other" (Kaminsky 1999, 100).

El migrante de Díaz se configura, siguiendo los planteamientos de Cornejo Polar (1996, 1995), como un sujeto migrante, un sujeto internamente heterogéneo que produce un discurso descentrado y doblemente situado. Este sujeto habla desde los diferentes lugares que coexisten en su memoria fragmentada, pero sin que estos desemboquen en un espacio armonioso. El constante ir y venir entre el aquí y ahora y el allá y ayer forma un "ritmo binario" (Kanellos 2011, 60, 166), un desdoblamiento del sujeto a través del cual las memorias le "siguen perturbando con rabia o con ternura" (Cornejo Polar 1995, 103). La percepción de algún sentimiento o acontecimiento sirve de mecanismo para reactivar la memoria del protagonista, como se observa, por ejemplo, en las repetidas referencias al sol, calor y, en particular, el hambre, elemento que según Ignizio $(2016,104-107)$ crea una asociación con la escasez de comida en el período especial en Cuba.

El sujeto migrante en esta novela se conforma como un sujeto individual, con unas experiencias migratorias singulares, pero en sus recuerdos reconstruye la historia de la Cuba revolucionaria, íntimamente entretejida con la historia de su familia. Esta historia se representa como una narrativa escindida entre el presente en Miami y el pasado en la isla, a la par que el protagonista de ahí plantea sus sueños del futuro. De hecho, la memoria de Stalin no se representa únicamente como un proceso interior, sino que en el presente narrativo algunos personajes se desdoblan como interlocutores, en particular la bella Miriam, la sobrina de su cuñada que le agrada con sus visitas a la azotea.

Haciendo referencia paratextual al título de la novela, Miriam repite en inglés "Tell me something about Cuba" (Díaz 1998, 78, 119). La petición no solo le obliga a Stalin a profundizar en la memoria y compartir sus recuerdos de la isla, con lo cual, como observa Gustafsson $(2014,169)$, la memoria trasciende lo individual, sino que también subraya la inquietud de la joven por conocer sus raíces culturales y, por ende, familiares: la idea de reconstruir en la imaginación esa patria tan cercana como distante para entender el pasado de su propia familia. Nacida en Cuba, pero criada en Estados Unidos, Miriam representaría lo que Pérez Firmat (2012, 3-6) en su ensayo sobre la cultura y la identidad cubano-americanas llama la generación 1.5. Ahora bien, en la novela de Díaz, esta posición resulta algo más problemática de lo que indica Pérez Firmat, sobre todo en cuanto a las asimetrías de poder. Miriam ni habla español, ni se identifica como cubana, pero tampoco se considera totalmente "american" (Díaz 
1998, 120), pues esta última posición de sujeto le está negada tanto por la sociedad angloamericana hegemónica como por otros grupos étnicos a través de las fronteras culturales y sociales (Díaz 1998, 120). La pregunta “I ask myself who am I. Can you tell me?" (Díaz 1998, 120), expresa no solo la búsqueda identitaria del personaje sino también la idea de que este vacío se pueda curar a través del encuentro con las raíces, valiéndose del motivo literario de descubrir su "yo verdadero" a través de la historia familiar, por muy engañosa que sea esta idea (cf. Merolla 2011). En cierta manera, Miriam, que intenta comunicarse con Stalin en spanglish, se representa como un sujeto diaspórico, pero aculturado, con unos fuertes deseos de vivir una "vida en el guión" (life on the hyphen) (Pérez Firmat 2012, 3-6). Quiere poder circular tanto en la cultura del exilio de los padres como en la nueva; no estar limitada al aferramiento a la cultura cubana prerrevolucionaria ni suprimir su otredad en relación con la cultura mayoritaria, sino navegar y traducir entre las dos en un espacio transnacional.

\section{La familia dentro y fuera: las fronteras del exilio}

En el despliegue de la narrativa de la familia transnacional, el concepto de frontera tiene un papel central. La frontera, tanto en su concepción material como en su concepción metafórica, se entiende en el presente trabajo como una línea que separa, pero también como un espacio intermedio (in-between space) de contacto y conflicto, así como un movimiento: el cruce mismo (Grossberg 1996, 91-92, Giménez 2007, 19-22). En su estudio de la función utópica del discurso revolucionario cubano y sus efectos de interpelación y subjetividad, Gustafsson (2014) destaca que el "proyecto nacional, social y utópico se constituye principalmente como una frontera que divide entre dentro y fuera (tanto del proyecto como de la nación), entre los que emigran y los que no, y que divide entre memorias de la Cuba del pasado, del presente y del futuro" (158). Siguiendo a Kaminsky $(1999,23,32)$, la nación se entiende como un espacio tanto físico-geográfico como simbólico-político, y la base de la identificación nacional como el sentido de pertenencia a un lugar, generalmente en combinación con el efecto de una persuasiva ideología nacional. No obstante, muchas veces es solo al salir del territorio nacional, o al cruzar la frontera del lugar que lo simboliza, la "patria chica", que el sujeto llega a la consciencia de la identidad como sujeto nacional (Kaminsky 1999, 28, 3334). Dicho de otra manera, "la identidad como sujeto nacional depende de la fricción y muchas veces está atada a una pérdida, falta o añoranza" (Kaminsky 1999, 29). ${ }^{4}$

La dinámica interna de la familia en la novela se plasma como una narrativa de des- y reintegración, en primer lugar, a través de las fronteras nacionales y políticas, pero también en articulación con las categorías de generación, género y sexualidad. En la familia de Stalin hay una larga historia de migración y activismo político: el padre era emigrante gallego y ponía "la misma pasión desmedida [...] en todos los actos centrales de su vida" (Díaz 1998, 27), tales como "[a]ñorar a su tierra lejana, integrarse en su isla de promisión, proteger a su esposa, educar a su prole y defender fervorosamente las ideas comunistas" (Díaz 1998, 27-28). Justamente la pasión revolucionaria parece ser lo que sirve de amalgama en la familia y mantiene a sus integrantes dentro de los confines de la nación. No obstante, cuando el hijo más exitoso opta por el exilio, la familia se divide en dentro y fuera tanto del proyecto utópico-político como del espacio nacional: "Durante años fueron una familia unida, apasionadamente revolucionaria, pero Lenin rompió la piña al colarse en la embajada del Perú y venirse a Miami por el puente de Mariel” (Díaz 1998, 66).

\footnotetext{
${ }^{4}$ Todas las traducciones son del autor.
} 
Tanto en esta cita como en la anterior, la distancia irónica con la que el narrador cuenta la historia familiar desde el punto de vista del protagonista expone la diferencia generacional con respecto a los padres y su fervor revolucionario. A diferencia de los que salieron del país en los primeros años de la Revolución, en el éxodo por el Mariel, estigmatizados tanto por el gobierno cubano como por los mass media en Estados Unidos, participaron personas de todas las clases sociales, no solo los pudientes o antirrevolucionarios (Simal 2018). También habían pasado más de veinte años con la Revolución, que supuestamente les había beneficiado, lo que, como narra Díaz en la novela, causó graves actos de repudio contra los emigrantes por parte del régimen castrista. El hecho de que el nombre del protagonista, al igual que los de sus hermanos Lenin y Stalina, cargara con el signo de la utopía bajo el que han sido criados, aun cuando quiere cambiar de nombre (Gustafsson, 2014, 170), refuerza esta diferencia. Seña de una intención didáctica en el texto de denunciar las aberraciones más graves del discurso y proyecto revolucionarios de los años 1980, el protagonista reitera desde el aquí y ahora en Miami su arrepentimiento no solo de haber roto todo contacto con Lenin y considerarlo como muerto a través de un pacto con Stalina sino también de haber participado en un acto público de odio contra los disidentes en la embajada. De tal manera quedan expuestos los efectos de las relaciones geopolíticas sobre la familia y las respuestas de los miembros ante esos procesos, pues como dice Stalina, "lo único que realmente valía la pena en este mundo era la familia; ella, a quien la vida se la había destrozado, lo sabía muy bien" (Díaz 1998, 201). Igual que en tantas otras familias afectadas por la polarización política de la época, un espacio transnacional se abre entre ellos, caracterizado por una incomprensión e incomunicación infranqueables.

En lugar de la trama tradicional de un proceso migratorio lineal de transición de un lugar a otro o un viaje circular, un periplo (Olsson, 2016), el protagonista traza un itinerario novedoso de ida y vuelta y otra vez ida que incluye la travesía por el mar en balsa, el regreso en avión y el cruce terrestre de la frontera entre México y Estados Unidos. El primer viaje, de apenas un par de días de duración, sirve en el relato como un simulacro de los procesos de exilio y "desexilio" — empleo aquí el término de Benedetti (1985) — con el fin de iniciar el viaje interior del protagonista y proporcionarle la experiencia de destierro y reterritorialización. Esta breve e insólita aventura le permite a Díaz le explorar los mecanismos del sueño americano, la reunificación familiar en el extranjero y la problemática del regreso a la isla bajo el régimen actual.

Víctima de un secuestro del ferry que le llevaría a su casa al otro lado de la bahía, el protagonista termina sin quererlo en Key West. A diferencia de los demás pasajeros, interpelados por el sueño americano, Stalin decide regresar a la isla y solo pide ver brevemente a Lenin. Esta decisión, se acuerda el protagonista repetidamente, se debe al deseo de regresar a su mujer, la joven bailarina Idalys, que, según cree, le espera fiel en casa como una Penélope. No obstante, siguiendo el concepto de desexilio, si el personaje comienza a evolucionar a través de la experiencia del "exilio" en Miami y regresa con el germen de una nueva "mirada bifocal" (Said 2000, 186), el lugar de origen también se transforma en su ausencia. De hecho, al regresar a La Habana, todo está cambiado: en el aeropuerto le reciben como héroe de la patria, pero pronto se da cuenta de que su mujer ya no es su mujer y que en su casa duerme otro hombre, pues como explica el narrador, Idalys concibe el extranjero "como una especie de paraíso situado en el más allá, desde el que nadie en su sano juicio regresaría a la isla" (Díaz 1998, 170). Tanto para Idalys como ante la ley, el exilio equivale simbólica y técnicamente a la muerte para Stalin y la viudez para ella (Díaz 1998, 173). 
Kaminsky $(1999,5-8)$ señala cómo uno de los motivos más persistentes en la literatura de exilio escrita por hombres es la representación simbólica de la patria como una figura femenina. En la novela de Díaz y el caso particular del exilio cubano, este nexo adquiere un sentido negativo. Con el padre fallecido y el hermano emigrado e incomunicado, a Stalin le queda un cuarteto de figuras femeninas significativas en una "idealización masculinista" (Kaminsky 1999, 7) de la mujer como nación: madre, hermana, sobrina y, en particular, esposa, puesto que en ella y en su engaño se centra la mayoría de los recuerdos. Ahora bien, quisiera plantear que la narrativa del fracaso del matrimonio está íntimamente entrelazada con la narrativa del desmoronamiento de la Revolución para el protagonista, un matrimonio que, además, no ha dado fruto, es decir, no ha contribuido a la reproducción de la nación. Posicionándose en el relato como el engañado, Stalin también se reconoce como víctima inocente de un proyecto social que para él se ha convertido en, para citar a Gustafsson (2014), "signo de privaciones y arbitrariedades" (170). De hecho, el engaño solo es el inicio de una cadena de humillaciones que tiene que sufrir al volver: sin casa, se ve obligado a regresar reaciamente al hogar maternal, pero solo para darse cuenta de que las autoridades han decidido cerrar el restaurante familiar que acaban de inaugurar por incompatibilidad con su nuevo estatus de héroe de la patria. A esto se puede añadir que Stalin se queda sin los objetos más significativos, tan vitales para su vida precaria que los convierte en miembros de la familia: el viejo ventilador Pepe, que se queda con Idalys, la antigua bicicleta Fredesvinda, que termina en la basura en Estados Unidos, y la nueva y flamante bici de montaña que su hermano le regala en Miami y que le roban la misma noche que descubre el engaño de su (ex)mujer.

Si el primer cruce de la frontera de dentrolfuera es como balsero involuntario y termina con el regreso al hogar maternal, el segundo, el paso clandestino de la frontera mexicanoestadounidense, es definitiva: no hay vuelta atrás. Cuando Stalin es enviado a México a un congreso de odontología como un premio por su heroísmo de regresar voluntariamente de Miami, todavía no ha tomado la decisión consciente de exiliarse, pero a través del viaje interior pronto llega a la consciencia de que la isla se ha convertido en un no-lugar para él: "sabía perfectamente que al regresar se encontraría sin mujer, sin casa, sin restorán, sin bicicleta y sin futuro" (Díaz 1998, 196). El viaje académico se convierte así en una complicada huida hacia el Norte, tópico común de la novela de la migración latinoamericana indocumentada a Estados Unidos ( $c f$. Olsson, 2016): "No tenía más alternativa que seguir huyendo hacia adelante" (Díaz 1998, 221). Aquí, la hermana tiene el rol de "catalizadora" de la entrada clandestina en Estados Unidos y, por tanto, contribuye a la dispersión espacial de la familia, lo que denota un cambio fundamental en relación con el exilio del hermano mayor años atrás y hace subrayar la diferencia de generación política.

\section{Entre el sueño del retorno y el sueño miamense}

Con este giro original de la trama, el protagonista ya no representa tanto la condición del exiliado — se halla en la azotea para aparentar balsero auténtico- sino que más bien ocupa el lugar del sujeto migrante indocumentado, figura ya tópica de la literatura mexicana, centroy sudamericana de la migración ( $c f$. Olsson 2016). Díaz explora la intersección entre migración y exilio, una categorización que muchas veces resulta artificial, pues ambos tipos de refugiado - económico y político - muchas veces coinciden en el mismo sujeto. Igual que el caso cubano, las otras migraciones latinoamericanas tienen elementos económicos y políticos, y las fronteras, sean por tierra o por mar, dividen la familia transnacional para el que no pueda salir o regresar. 
Al hallarse de nuevo en Estados Unidos, el personaje, escondido en el maletero del coche de un policía estadounidense, se da cuenta de que la entrada no autorizada es solo un primer paso para el migrante irregular: la frontera sigue multiplicándose al interior de la nación. El pasaje en el aeropuerto de Brownsville, donde va a tomar un vuelo doméstico a Miami, recuerda el miedo omnipresente de vivir como indocumentado. Las historias y formas de llegar condicionan la orientación del sujeto, sostiene Ahmed (2006, 10). Si describimos la migración como un proceso de desorientación y reorientación, podemos entender la orientación del sujeto migrante como la experiencia vivida de encarar por lo menos dos orientaciones: hacia una patria/hogar que se ha perdido y hacia un lugar que el sujeto todavía no habita, es decir, que todavía no se ha convertido en "casa" (Ahmed 2006, 9-10). La azotea, imaginada por el protagonista en términos marítimos como una "balsa" (Díaz 1998, 219), está a-isla-da tanto del resto de la vivienda familiar como de la sociedad que le rodea. Se rige por su propio tiempo, que obedece al paso del sol, el hambre, la sed, las visitas de Miriam y la cuenta atrás para el día de su entrega a las autoridades estadounidenses, marcada por los epígrafes de los capítulos. La mirada del protagonista se convierte en un motivo recurrente de la doble orientación: o bien queda fijada "hacia el sur, hacia la inalcanzable Cuba" (Díaz 1998, 24), o bien hacia "el ajeno perfil de Miami” (Díaz 1998, 26), donde el personaje una y otra vez contempla "los lejanos rascacielos del downtown" (Díaz 1998, 24) y los coches que circulan por el expressway (Díaz 1998, 65, 104-105), símbolos de libertad y de la modernidad capitalista que le produce extrañamiento.

A la espera de conquistar esa libertad, es decir, ocupar y habitar el nuevo lugar, el encierro en la azotea representa para el personaje un purgatorio donde tiene que encarar una condena tripartita que va transformando su cuerpo. Primero, es posible entender las marcas en la piel que dejan el sol, el hambre y la sed como una condena por haber entrado en Estados Unidos sin autorización, ya que esto le obliga a convertirse en falso balsero para legalizar más fácilmente su estancia. Segundo, como sostiene Gustafsson $(2014,158)$ en su análisis del discurso utópico en la Cuba revolucionaria, el que se va de la isla se convierte en "cobarde" y "traidor", porque no solo abandona "el buen lugar" sino también su compromiso con el futuro de la patria. A lo largo de la narración, el protagonista hace hincapié en la "culpa del exilio" (Díaz 1998, 124), pero en esta novela se trata menos de haber abandonado el proyecto nacional que de haber dejado a los seres queridos: "El olor a su madre, de su infancia y de su lengua habían quedado atrás para siempre, pero los suyos seguían allá, prisioneros de aquel mundo sin esperanza, y frente a ellos se sentía un traidor" (Díaz 1998, 124). Relacionada con la frontera de dentrolfuera está la frontera política que ha obligado a los hermanos a la separación e incomunicación y que el protagonista percibe como "una cuenta inexplicable que saldar" (Díaz 1998, 249). De hecho, la idea de Lenin de convertir a su hermano en "falso balsero" puede verse como una venganza (in)consciente por haber roto el contacto con él durante los años de exilio. A pesar de estar configurada como un no-lugar, la azotea sobresale como un espacio significativo en el que la dinámica interna de la familia se entreteje con las relaciones externas de sus miembros.

La orientación dual del migrante es el punto de partida del proceso de habitar el nuevo espacio (Ahmed 2006, 10). Si retomamos los planteamientos de Kaminsky (1999, 6-8) sobre la intersección entre migración, género y nación, podemos advertir que el recurso para marcar la orientación del sujeto migrante es la narrativa de sus relaciones amorosas. Con la entrada de Miriam en la historia y el espacio de la azotea, cambia tanto el objeto del amor de Stalin como sus recuerdos y la relación con ella puede entenderse como un intento de dar sentido a su 
existencia fragmentada, pues como afirma Kaminsky, en la literatura del exilio escrita por hombres, el "exilio desequilibra, desestabiliza el espacio, pero los relatos familiares de heterosexualidad anclan las historias" $(1999,55)$. Siguiendo la dualidad tradicional sexista de virgen/puta, Miriam aparece con toda su bondad como una figura angelical en el purgatorio de la terraza, mientras que el protagonista se acuerda de su (ex)esposa como engañosa, igual que la desilusión que siente ante la utopía de la Revolución: "El recuerdo de Idalys era amargo y le provocaba deseos de venganza; el de Miriam, en cambio, era dulce como la caña de azúcar" (Díaz 1998, 181). La unión sexual entre Stalin y Miriam representa para él un rito de iniciación en la nueva sociedad o, como lo expresa Kaminsky, "adaptación por coito intercultural" (1999, 53), mientras que para ella simboliza el deseo de encontrarse con sus raíces culturales. Sin embargo, a diferencia del patrón tradicional, esta relación no es ni endógama ni exógama, sino que la ambivalente posición de Miriam como insider/outsider en Miami —un espacio transnacional de contacto y conflicto - de alguna manera refracta la posición del protagonista. En la imaginación del protagonista, el sexo de Miriam, "abierto como las puertas del paraíso" (Díaz 1998, 181), queda también abierto a la interpretación: puede significar la conquista del nuevo espacio y la entrada en la diáspora, pero por las alusiones a la geografía cubana también puede ser una metáfora del regreso a una isla libre, ya que el personaje piensa que "quizá, al recibir el beso, despertaría amándola en una Cuba tranquila, sin revolución y sin exilio" (Díaz 1998, 228-229).

Ante la doble orientación del sujeto migrante y la separación de la familia por la frontera dentrolfuera, el protagonista queda en la posición ambivalente que se ve aludida por sus sueños con la joven cubano-americana. Por un lado, la nostalgia, que no le deja dormir en paz (Díaz 1998, 150), y el sueño del retorno (Kanellos 2011), según el que se ve de regreso en la isla con Miriam después de la muerte de Fidel. Por otro, el "sueño miamense", que no solo consta de la utopía de un exilio consumista (Gustafsson 2014, 169), centrada en la idea de abrir su propia clínica, sino también del sueño de una familia unida:

\begin{abstract}
el sueño también podía buscarse en el mañana, en la ilusión de llegar a tener su clínica y de reconciliarse con Leo en una fiesta en la que se harían una foto de familia junto a sus respectivas mujeres, a su madre y a Stalina [...] a sacar a Idalys de la foto y a introducir en su lugar a Miriam, que ahora sonreía junto a él y era su esposa [...] empezó a sonreír y estuvo haciéndolo hasta que se estrelló contra la evidencia de que los personajes del retrato no vivían en la misma ciudad ni en el mismo país y que no había cámara en el mundo capaz de reunirlos. (Díaz 1998, 247)
\end{abstract}

Por medio del motivo de la foto familiar, emblema de la felicidad familiar, el protagonista imagina la reunificación. Con la resolución del triángulo amoroso cuando un emparejamiento heterosexual es cambiado por otro, Stalin echa a su exmujer de la foto para incluir a Miriam y con eso se deshace de la figura principal de la patria. Sin embargo, los sentimientos cambiantes del protagonista hacen ver las grietas en el sueño: la familia unida no es sino otra utopía del exiliado.

La escena final refuerza esta ambivalencia acerca del futuro del indocumentado. A pesar de los peligros evidentes, Stalin insiste en llevar a cabo el plan: poner a la deriva una auténtica balsa en medio del mar, para que luego aparezca con él en la playa. De tal manera comienza una nueva separación espacial entre los hermanos, pero una conmovedora escena del perdón simboliza la reunificación emocional por encima de la frontera política. No obstante, la idea disparatada de salir en una balsa una noche de mar grueso y sin saber navegar amenaza con hacer la separación definitiva, y esta incertidumbre se subraya por la última página en blanco, 
que solo lleva el título "Martes 28" (Díaz 1998, 261). Este silencio significativo deja abierto a la imaginación el final del proceso migratorio del protagonista, pero si retomamos la conceptualización de la frontera como un espacio de conflicto y contacto, podemos advertir que la hibridación cultural y lingüística del espacio transnacional a la que se alude en el texto parece indicar otra posibilidad. De la misma manera que el spanglish que les acerca a Stalin y Miriam, franqueando las fronteras lingüísticas y generacionales (Díaz 1998, 120), la bandera estadounidense en el yate del contrabandista que le lleva al mar y la leyenda inscrita en la popa "Cubita bella. Miami” (Díaz 1998, 260) consisten en la yuxtaposición de elementos aparentemente irreconciliables, según el discurso oficial de dentrolfuera, en un espacio fronterizo que no solo divide, sino que también reúne. Parece que, para Díaz, el futuro del exilio y la diáspora cubanos reside en el perdón, el acercamiento y el diálogo cultural por sobre las fronteras. Ello podría unir a las familias escindidas, aunque no siempre reunificarlas físicamente.

\section{Conclusiones}

Este estudio se ha centrado en la representación literaria de la familia transnacional en Dime algo sobre Cuba. La novela se posiciona en la intersección entre exilio, diáspora y migración indocumentada en el contexto del período especial de los años 1990. Hemos observado cómo la dinámica interna de la familia está íntimamente entretejida con las relaciones externas con los dos lugares principales del mundo narrado, La Habana y Miami, sinécdoques de sus respectivas naciones. Una particularidad de lo local/cubano en esta narrativa de la familia transnacional es el discurso utópico-revolucionario, que no solo marca la frontera entre dentro y fuera del proyecto y de la nación, sino también divide a las familias ya antes de emigrar. Esta frontera hace resaltar la separación, la exclusión y la incomunicación como aspectos especialmente desgarradores de la familia transnacional cubana. Hasta cierto punto, la representación espacial sigue las pautas establecidas de la literatura del exilio escrita por hombres: la patria se imagina como un cuerpo femenino, mientras que el exilio se configura como una posición masculina, aunque aquí la feminización de la nación de origen cobra un sentido negativo de traición y decepción. En específico, la orientación del migrante es simbolizada por el emparejamiento heterosexual, de manera que la resolución del triángulo amoroso conlleva el cambio del sentido de pertenencia del protagonista y su distanciamiento final del proyecto revolucionario. No obstante, también hemos señalado cómo el proceso migratorio del protagonista traza un insólito itinerario de ida y vuelta y otra vez ida que forma un nexo con la literatura de la migración latinoamericana indocumentada a Estados Unidos. A través de este se pone de manifiesto la posición ambivalente del personaje entre el exilio y el desexilio, así como su condición vulnerable de indocumentado, aislado tanto del resto de la familia como de la sociedad que le rodea por una frontera política, cultural y social que se multiplica dentro del territorio estadounidense. A pesar del final abierto, la hibridación del espacio transnacional, que incluye el acercamiento de elementos heterogéneos y aparentemente incompatibles, indica una posible apertura hacia el futuro para la (re)integración y la reunificación familiar.

\section{Bibliografía}


Ahmed, Sara. 2006. Queer Phenomenology: Orientations, Objects, Others. Durham y Londres: Duke University Press.

Aínsa, Fernando. 1988. "La alteridad lejana como utopía en el mito de la tierra prometida". Cuadernos Americanos 10: 55-80.

Anderson, Benedict. 2006 [1983]. Imagined Communities: Reflections on the Origin and Spread of Nationalism. Londres: Verso.

Benedetti, Mario. 1985. El desexilio y otras conjeturas. Madrid: Ediciones El País.

Bryceson, Deborah, y Ulla Vuorela. 2002. "Transnational Families in the Twenty-first Century". En The Transnational Family: New European Frontiers and Global Networks, editado por Deborah Bryceson y Ulla Vuorela, 3-30. Londres: Bloomsbury Publishing.

Cornejo Polar, Antonio. 1995. “Condición migrante e intertextualidad multicultural: El caso de Arguedas”. Revista de Crítica Literaria Latinoamericana 21 (42): 101-109.

Cornejo Polar, Antonio. 1996. "Una heterogeneidad no dialéctica: Sujeto y discurso migrantes en el Perú moderno". Revista Iberoamericana LXII (176-177): 837-844.

Díaz, Jesús. 1998. Dime algo sobre Cuba. Madrid: Espasa.

Dreby, Joanna, y Timothy Adkins. 2010. "Inequalities in Transnational Families". Sociology Compass 4 (8): 673-689.

Eckstein, Susan, y Lorena Barberia. 2002. "Grounding Immigrant Generations in History: Cuban Americans and Their Transnational Ties". The International Migration Review 36 (3): 799-837.

Giménez, Gilberto. 2007. "La frontera norte como representación y referente cultural en México". Cultura y representaciones sociales 2 (3): 17-34.

Goulborne, Harry, Reynolds Tracey, John Solomos, y Elisabetta Zontini. 2010. Transnational Families: Ethnicities, identities and social capital. Londres y Nueva York: Routledge.

Grenier, Yvon. 2017. “Jesús Díaz, 1941-2002. The Unintentional Deviationist”. Cuban Studies 45: 115-131.

Grossberg, Lawrence. 1996. "Identity and Cultural Studies - Is That All There Is?”. En Questions of Cultural Identity, editado por Stuart Hall y Paul du Gay, 87-107. Londres: SAGE.

Guerrero, Gustavo. 2002. “Jesús Díaz: ilusión y desilusión”. Encuentro de la Cultura Cubana 25 (invierno): 1-18.

Gustafsson, Jan. 2014. "Entre el buen lugar y el no-lugar. Utopía, memoria y migración en la Cuba revolucionaria". Meridional. Revista Chilena de Estudios Latinoamericanos 3: 153-174.

Ignizio, Graham. 2016. "Food, Memory, and a Starving Dentist: Jesús Díaz's Special Period in Times of Peace". Studies in Latin American Popular Culture 34: 96-108.

Kaminsky, Amy K. 1999. After Exile: Writing the Latin American Diaspora. Minneapolis: University of Minnesota Press.

Kanellos, Nicolás. 2011. Hispanic Immigrant Literature: El Sueño del Retorno. Austin: University of Texas Press.

Martínez Ruiz, Diana Tamara, y Miriam Reyes Tovar. 2017. “Un acercamiento etnográfico a la cotidianidad de las dinámicas familiares en un contexto de migración internacional 
México-Estados Unidos”. En Hogares y familias transnacionales. Un encuentro desde la perspectiva humana, editado por J. Guadalupe Rodríguez Gutiérrez, Miguel Moctezuma Longoria y Óscar Calderón Morillón, 109-135. México, D.F.: Universidad de Sonora, Benemérita Universidad Autónoma de Puebla, Juan Pablos Editor.

Merolla, Daniela. 2002. "Deceitful Origins and Tenacious Roots: Moroccan Immigration and New Trends in Dutch Literature". En The Transnational Family: New European Frontiers and Global Networks, editado por Deborah Bryceson y Ulla Vuorela, 103123. Oxford: Berghahn.

Olsson, Fredrik. 2016. "Me voy pal Norte”. La configuración del sujeto migrante en ocho novelas hispanoamericanas actuales (1992-2009). Madrid: Consejo Superior de Investigaciones Científicas, Diputación de Sevilla, Universidad de Sevilla.

Pérez Firmat, Gustavo. 2012. Life on the Hyphen: The Cuban-American Way [2a ed.] Austin: University of Texas Press.

Rojas, Rafael. 2006. Tumbas sin sosiego. Revolución, disidencia y exilio del intelectual cubano. Barcelona: Anagrama.

Said, Edward W. 2000. Reflections on Exile and Other Essays. Cambridge, Massachusetts: Harvard University Press.

Sánchez Becerril. 2012. "Consideraciones teórico-críticas para el estudio de la narrativa cubana del periodo especial”. Literatura: teoría, historia, crítica 14 (2): 83-112.

Simal, Mónica. 2018. "Narrar a Mariel. Espacialización y heterotopías del exilio cubano en la novela Boarding home (1987), de Guillermo Rosales (1946-1993). Latin American Research Review 53 (2): 318-329.

Sommer, Doris. 1991. Foundational Fictions: The National Romances of Latin America. Berkeley, Los Ángeles y Oxford: University of Califo 